\title{
Sciences du jeu
}

2| 2014

Questionner les mises en forme ludiques du web : gamification, ludification et ludicisation

\section{Du game design au gamefulness : définir la gamification}

Sebastien Deterding, Dan Dixon, Rilla Khaled et Lennart Nacke

\section{OpenEdition}

\section{Journals}

Édition électronique

URL : http://journals.openedition.org/sdj/287

DOI : $10.4000 /$ sdj.287

ISSN : 2269-2657

Éditeur

Laboratoire EXPERICE - Centre de Recherche Interuniversitaire Expérience Ressources Culturelles Education

\section{Référence électronique}

Sebastien Deterding, Dan Dixon, Rilla Khaled et Lennart Nacke, « Du game design au gamefulness : définir la gamification », Sciences du jeu [En ligne], 2 | 2014, mis en ligne le 24 octobre 2014, consulté le 27 mars 2021. URL : http://journals.openedition.org/sdj/287 ; DOl : https://doi.org/10.4000/sdj.287

Ce document a été généré automatiquement le 27 mars 2021

\section{(c) (i) (9)}

La revue Sciences du jeu est mise à disposition selon les termes de la Licence Creative Commons Attribution - Pas d'Utilisation Commerciale - Pas de Modification 4.0 International. 


\title{
Du game design au gamefulness : définir la gamification
}

\author{
Sebastien Deterding, Dan Dixon, Rilla Khaled et Lennart Nacke
}

\section{NOTE DE L'ÉDITEUR}

Ce texte est la traduction par Antoine Dauphragne de « From Game Design Element to Gamefulness: Defining "gamification" ", Proceeding MindTrek '11 Proceedings of the 15th International Academic MindTrek Conference: Envisioning Future Media Environments, Tampere, Finland - 28-30 septembre, 2011, téléchargeable à l'adresse suivante: http://85.214.46.140/niklas/bach/

MindTrek_Gamification_PrinterReady_110806_SDE_accepted_LEN_changes_1.pdf Un certain nombre de terme ne pouvant être traduits de l'anglais au français sans perdre leur logique conceptuelle, nous avons donc fait le choix de maintenir dans un certain nombre de cas les termes anglais originaux, en particulier du fait de l'existence de deux termes pour désigner en anglais le jeu, game et play.

\section{Introduction}

1 Suite au succès du service de géolocalisation Foursquare, l'idée d'utiliser des éléments de game design dans des contextes non ludiques pour motiver et améliorer l'activité et la fidélité des utilisateurs a rapidement gagné du terrain dans les domaines de la conception d'interfaces et du markéting numérique. Sous l'appellation "gamification ", cette idée engendre un intense débat public ainsi que de nombreuses applications allant de la productivité à la finance, en passant par la santé, l'éducation et le développement durable, jusqu'aux médias de l'information et du divertissement. Plusieurs prestataires vendent maintenant comme "gamification» une couche logicielle de service proposant des systèmes de récompense et de réputation avec des points, des badges, des niveaux et des classements. Ce déploiement commercial d'applications « gamifiées » auprès d'un large public laisse potentiellement augurer de 
nouvelles pistes de recherche intéressantes ainsi que de nouvelles sources de données pour l'étude de l'interaction homme-machine (IHM) et pour les études sur le jeu - et, en effet, la gamification retient de plus en plus l'attention des chercheurs (Deterding et al, 2011 ; McGonigal, 2011 ; Reeves \& Read, 2009).

Cependant, jusqu'à maintenant, peu de travaux académiques se sont penchés sur la définition du concept de "gamification » (à l'exception de Huotari \& Hamari, 2011). De même, la question de savoir si le terme renvoie bien à un phénomène suffisamment nouveau et distinct n'a pas fait l'objet d'un examen minutieux. C'est pourquoi cet article recense et situe les usages actuels du mot «gamification » dans les recherches existantes sur le sujet, afin de proposer une définition de la gamification. Les premières parties de ce travail décrivent l'origine et les usages actuels du terme et les comparent avec leurs précurseurs historiques ainsi qu'avec des termes alternatifs dans les domaines de l'IHM et des études sur le jeu. Cela mène à une définition de la gamification et à une discussion sur les éléments qui la composent. Nous soutenons que la gamification met en lumière des phénomènes de gamefulness, qui devraient être considérés à la fois comme complémentaires et distincts des phénomènes de playfulness. Après avoir situé notre définition dans les champs de l'IHM et des études sur le jeu, l'article conclut en mettant en avant la contribution à la recherche que représente l'étude des applications « gamifiées ».

\section{Aux origines : I'industrie du numérique}

3 Le terme " gamification » a été forgé dans l'industrie des médias numériques. Le premier usage référencé remonte à 2008 (Paharia, 2010 ; Reeves \& Read, 2009), mais le terme ne fut pas pleinement adopté avant la seconde moitié de l'année 2010. Des termes concurrents continuent à être utilisés, et de nouveaux sont toujours introduits; on peut citer productivity games (McDonald, Musson \& Smith, 2008), surveillance entertainment (Grace \& Hall, 2008), funware (Takahashi, 2008), playful design (Ferrara, 2012), behavioral games (Dignan, 2011), game layer (Priebatsch, 2011) ou applied gaming (natronbaxter.com). Pourtant, "gamification» s'est peu à peu institutionnalisé comme le terme le plus commun.

4 En dépit ou à cause de cela, "gamification » est aussi un terme fortement contesté, en particulier au sein de l'industrie du jeu et de la communauté des études sur le jeu. L'insatisfaction devant les façons de mettre en œuvre ce concept, les simplifications abusives et les interprétations dont il fait l'objet ont conduit certains à forger des termes différents pour leurs propres travaux dans des domaines très proches. Par exemple, la chercheuse et designer Jane McGonigal (2011, p. 120) a redéfini « jeux en réalité alternée » comme « un jeu auquel on joue dans la vie réelle » pour décrire son travail, et le spécialiste du jeu et designer Ian Bogost (2011) a recommandé de remplacer le terme "gamification» par «exploitationware» afin de signifier un acte politico-linguistique qui décrirait avec plus d'acuité le « règne infâme de la tromperie » que la gamification est supposée instaurer.

5 Les usages actuels du terme dans l'industrie fluctuent entre deux concepts liés entre eux. Le premier renvoie à l'adoption, l'institutionnalisation et l'omniprésence croissantes des jeux (vidéo) dans la vie de tous les jours (Schell, 2010 ; Helgason, 2010 ; Chatfield, 2005). La seconde notion, plus spécifique, avance que puisque l'objectif premier des jeux vidéo est le divertissement et puisqu'ils peuvent motiver leurs 
utilisateurs à s'y engager avec une intensité et une durée sans équivalent, les éléments ludiques devraient aussi être capables de susciter plus de plaisir et d'engagement dans des produits et des services qui ne sont pas des jeux (Zichermann \& Cunningham, 2011; Flatla et al, 2011). strie du jeu" et des méthodes du énéfices clientsPrestataires et consultants ont eu tendance à décrire la gamification de manière pratique et en termes de prestations aux clients, par exemple comme «l'adoption de la technologie du jeu et des méthodes du game design en dehors de l'industrie du jeu » (Helgason, 2010), « le fait de penser comme dans un jeu et d'utiliser des mécanismes de jeu pour résoudre des problèmes et intéresser les utilisateurs " (Zichermann, 2011) ou comme "l'intégration de dynamiques de jeu dans votre site, service, communauté, contenu ou campagne, afin d'entraîner la participation ${ }^{1}{ }^{1}$

\section{Concepts précurseurs et parallèles}

6 Ces idées ne sont pas entièrement nouvelles. La notion selon laquelle le design de l'interface utilisateur peut être façonné par d'autres pratiques de design possède une riche tradition en IHM. Durant le premier boom des jeux vidéo au début des années 1980, Malone a écrit des articles fondateurs tirant des jeux vidéo « une heuristique pour développer des interfaces divertissantes pour les utilisateurs" (Malone, 1981). L'analyse du design des premiers jeux d'aventure textuels tels qu'Adventure a conduit Carroll (Carroll, 1982), avec Thomas (Carroll \& Thomas, 1982), à suggérer d'améliorer les activités de travail routinières en utilisant diverses « fictions métaphoriques » afin qu'elles soient intrinsèquement plus intéressantes et d'appeler avec insistance à un programme de recherche sur le divertissement et sa propension à faciliter les usages (Carroll \& Thomas, 1988).

7 Avec l'expansion et la maturation du champ ainsi qu'avec l'émergence de l'expérience de l'utilisateur dans la sphère professionnelle, davantage de chercheurs ont entrepris d'étudier de tels "attributs hédoniques» (Hassenzahl, 2003) ou "affordances motivationnelles» (Zhang, 2008) de "produits agréables», qualifiant le champ de " "funology" - la science de la technologie divertissante» (Blythe, Hassenzahl \& Wright, 2004) et prenant à nouveau le game design comme une importante source d'inspiration. Dans cette mouvance, certains chercheurs ont étudié les "jeux à objectif ", dans lesquels le game play est détourné pour résoudre des tâches d'information telles que le marquage d'images (Ahn \& Dabbish, 2008), et qui utilisent des interfaces et des contrôleurs de jeu dans d'autres contextes (Chao, 2001). Surtout, de nombreux chercheurs ont exploré le concept de playfulness comme une expérience d'utilisateur ou un mode d'interaction désirable ainsi que les modalités de design qu'il implique. En dépit de ce considérable corpus de recherche, aucune théorie ou terminologie consensuelle de "playfulness " n'a encore émergé. Parfois, on en fait vaguement l'équivalent de n'importe quelle «expérience agréable" (Costello \& Edmonds, 2007), ou de "divertissement» (Fontijn \& Hoonhout, 2007), ou de toute interaction qui va au-delà des contextes du travail et des tâches utilitaires (Gaver et al, 2004 ; Gaver, 2002 ; Morrison, Mitchell \& Brereton, 2007). Pour pallier ce manque, Gaver a introduit les termes «design ludique», «engagement ludique» et "activités ludiques", qui décrivent grosso modo "les activités motivées par la curiosité, l'exploration et la réflexion» (Gaver et al, 2004). D'autres études ont ciblé et défini le concept de playfulness plus précisément (Woszczynski, Roth \& Segars, 2002 ; Morrison, 
Viller \& Mitchell, 2011 ; Korhonen, Montola \& Arrasvuori, 2009). Korhonen, Montola et Arrasvuori ont fait les tentatives les plus systématiques en la matière (Korhonen, Montola \& Arrasvuori, 2009; Lucero \& Arrasvuori, 2010) : en combinant le cadre de "l'expérience agréable» de Costello et Edmonds (Costello \& Edmonds, 2007) avec d'autres références théoriques et études sur les utilisateurs dans le domaine du jeu vidéo, ils ont développé un cadre de l'expérience playful ou PLEX (Playful Experience Framework) qui catégorise 22 expériences de ce type (une première version de ce travail en répertoriait 20).

Finalement, dans les années 2000, des chercheurs en IHM ont également montré un intérêt croissant pour l'étude du design et de l'expérience induite par les jeux vidéo eux-mêmes, en développant des méthodes pour évaluer l'expérience vécue par leurs utilisateurs (Bernhaupt, 2010), une heuristique de la « jouabilité » pour leur conception (Schaffer, 2008) ainsi que des modèles pour les composantes des jeux (Fullerton, 2008; Hunicke, Leblanc \& Zubek, 2004) et l'expérience de jeu (Calvillo-Gamez, Cairns \& Cox, 2010 ; Nacke, Drachen \& Goebel, 2010 ; Sweetser \& Wyeth, 2005).

Dans le champ des études sur le jeu, la gamification peut être considérée comme une excroissance de plus dans la réutilisation et l'extension des jeux au-delà du divertissement dans la sphère privée.

L'usage de jeux à des fins sérieuses, ce que l'on appelle aujourd'hui serious games, est vieux de plusieurs millénaires (Abt, 1970) et a évolué, passant d'usages principalement militaires à des usages touchant l'éducation et le milieu des affaires dans la seconde moitié du 20e siècle. Au début des années 2000 , le développement des jeux numériques a revigoré cette tendance en une industrie substantielle et en un champ de recherche spécifique. On peut définir ces jeux numériques sérieux comme "toute forme de logiciel de jeu interactif conçu pour être utilisé par un ou plusieurs joueurs sur toute plateforme et qui a été développé dans l'intention de proposer davantage que du simple divertissement (Ritterfeld, Cody \& Vorderer, 2009, p. 6). Dans le champ des serious games, certains auteurs ont proposé de différencier "serious games" et "serious gaming " (Jenkins et al, 2009). Alors que le terme «serious games » désigne des jeux développés pour véhiculer du contenu éducatif pendant qu'on y joue, "serious gaming » englobe toute utilisation (éducative) du jeu au sens large - c'est-à-dire toutes les technologies, pratiques, connaissances et tous les processus sociaux entourant les jeux, comme la critique de jeux, la production de machinimas, la conception d'objets, d'avatars, de niveaux virtuels, voire de jeux entiers.

11 Parallèlement au mouvement des serious games, de nouveaux genres de jeu ont évolué jusqu'à étirer les limites traditionnelles entre les jeux, amenant ces derniers vers de nouveaux contextes, de nouvelles situations, de nouveaux espaces. Généralement nommés "jeux pervasifs", ces jeux possèdent "une ou plusieurs caractéristiques saillantes qui étendent le cercle magique contractuel $\mathrm{du}$ jeu spatialement, temporellement et socialement » (Montola, Stenros \& Waern, 2009, p. 12). On en trouve des exemples parmi les jeux de géolocalisation qui déplacent le gameplay dans l'espace public, les jeux en réalité augmentée qui utilisent des outils numériques pour recouvrir l'environnement de représentations du jeu, les jeux persistants qui fonctionnent continuellement de manière à ce qu'on puisse y entrer et sortir à n'importe quel moment de la journée et les jeux en réalité alternée qui « s'emparent de la substance de la vie de tous les jours pour la tisser en trames narratives qui superposent de nouvelles 
couches de sens, ajoutent de la profondeur et des niveaux d'interaction au monde réel » (Montola, Stenros \& Waern, 2009, p. 37).

12 À plus grande échelle, les chercheurs spécialistes des médias observent une «ludification de la culture» (Montola, Stenros \& Waern, 2009; Raessens, 2006). Ils avancent que les jeux vidéo, par leur omniprésence, leur diffusion et leur institutionnalisation croissantes lors des trois dernières décennies, sont devenus un médium culturel et une source d'expériences formatives comme l'ont été la littérature, le cinéma ou la télévision pour les générations précédentes. Les technologies, les tropes, les références et les métaphores, les mentalités et les pratiques dérivant des jeux imprègnent de plus en plus la société et la vie de tous les jours, en particulier les identités et les pratiques médiatiques que l'on qualifiera de " playful».

\section{Vers une définition}

Cette brève revue de littérature montre que la gamification s'est développée sur un terrain fertile de tendances et de traditions en interaction dans les domaines du design numérique et des jeux et qu'il existe déjà un certain nombre de concepts qui peuvent lui faire concurrence, coexister avec elle ou lui être superposés. Par conséquent, si l'on veut comprendre et développer la "gamification" en tant que terme académique, notre tâche est de déterminer si le terme et les applications " gamifiées » actuelles sont significativement différents de phénomènes et de domaines de recherche antérieurs et, si c'est le cas, de les situer par rapport à ces champs préexistants.

Nous pensons que la gamification délimite bel et bien un ensemble de phénomènes distincts mais auparavant non identifiés, à savoir le complexe des concepts de gamefulness, gameful interaction et gameful design, qui diffèrent des concepts plus établis que sont playfulness, playful interaction ou design for playfulness. En considérant cette observation, nous proposons la définition suivante : la gamification est l'usage d'éléments de game design dans des contextes non ludiques. Les sous-parties suivantes développent cette définition en détail.

\subsection{Game}

En premier lieu, la gamification renvoie aux games, et non au play (ou au concept de playfulness), lequel peut être compris comme une catégorie plus large et plus souple, qui contient les games tout en étant différente d'eux (Salen \& Zimmerman, 2004). Dans les études sur le jeu, cette distinction entre play et games est habituellement adossée à la conception de Caillois de la paidia et du ludus comme deux pôles des activités ludiques (Caillois, 1958). Alors que la paidia (ou playing) désigne une recombinaison plus libre, plus expressive et même plus «tumultueuse » d'attitudes et de significations, le ludus (ou gaming) s'empare du playing en le structurant par des règles et un principe compétitif orienté vers des buts. Dans ce sens, les définitions classiques dans les études sur le jeu établissent que le gaming et les jeux - par opposition au playing et aux jouets sont caractérisés par des systèmes de règles explicites animés par une compétition ou une lutte dont les acteurs poursuivent des objectifs propres et obtiennent des résultats distincts (Juul, 2005; Salen \& Zimmerman, 2004). De récentes études théoriques et empiriques ont apporté d'autres ressources pour distinguer playing et gaming comme deux modes, pôles ou «valeurs » d'attitudes et d'états d'esprit ${ }^{2}$ rencontrés lorsqu'on 
joue à un jeu vidéo (Barr, 2007 ; Juul, 2010). Cette distinction apparaît aussi dans la recherche en IHM sur la notion de playfulness. Le cadre PLEX, que nous avons déjà mentionné, reconnaît la distinction de Caillois entre paidia et ludus en ce sens qu'il entreprend explicitement de saisir toute expérience entre ces deux pôles (Korhonen, Montola \& Arrasvuori, 2009). Enfin, tant dans le monde de la recherche que de l'industrie, les critiques des applications gamifiées ont souligné avec insistance leur focalisation presque exclusive sur les éléments favorisant le jeu de règles orienté vers un but (c'est-à-dire le ludus), laissant très peu de place pour le jeu ouvert, exploratoire et libre (c'est-à-dire la paidia) (Alfrink, 2011 ; Deterding, 2012). En effet, cette critique des applications gamifiées grand public apparaît comme une observation utile du point de vue de la recherche : cette conception inspirée par les games peut ainsi permettre des expériences et des attitudes penchant plus ou moins vers l'un ou l'autre pôle du jeu. Ces applications nous fournissent aussi des données empiriques sur le développement et l'expérience des systèmes basés sur les règles et le pôle du ludus, qui ont sans doute moins retenu l'attention des chercheurs en IHM.

Sur cette base, par opposition au cadre PLEX qui regroupe à la fois le jeu libre et le jeu de règles sous le terme "playfulness », nous suggérons d'adopter le terme

"gamefulness» récemment introduit par McGonigal (2011) comme complément systématique à "playfulness». Alors que "playfulness» fait référence d'une façon générale aux aspects expérientiels et comportementaux du jeu (paidia), " gamefulness " désigne les aspects relatifs aux games (ludus). Par conséquent, la notion de gamefulness circonscrit un ensemble cohérent de phénomènes spécifiques qui jusqu'à maintenant n'a pas fait l'objet d'un examen soutenu, nous laissant ainsi un terrain d'extension pertinent pour définir "gamification». Pour systématiser la terminologie, on peut distinguer :

- Gamefulness (l'aspect expérientiel et comportemental),

- Gameful interaction (les artefacts donnant accès à ces aspects),

- Gameful design (le fait de concevoir pour obtenir ces aspects, habituellement en utilisant des éléments de game design).

L'adoption de cette terminologie implique, en ce qui concerne la définition de "gamification», que celle-ci doit aussi être distinguée sur le plan analytique de " playfulness » et " playful design » - en effet, cette terminologie marque la nouveauté des applications gamifiées. En pratique, on peut supposer qu'elles vont souvent aussi susciter des attitudes et des états d'esprit playful, tout comme les joueurs de jeu vidéo oscillent souvent entre attitudes et états d'esprit playful et gameful quand ils jouent (Barr, 2007). «Gamification » coïncidera la plupart du temps avec « gameful design» tel qu'on l'a défini plus haut: la stratégie la plus probable pour concevoir des expériences de type gameful est d'utiliser des éléments de game design et l'objectif le plus probable lorsqu'on utilise des éléments de game design est de susciter ce type d'expérience. Pourtant sur le plan analytique, "gameful design» et "gamification» envisagent la même extension de phénomènes à travers différentes intentions - tels que la stratégie de développement consistant à utiliser des éléments de game design (gamification) ou l'objectif de développement visant à concevoir un produit dans une optique de gamefulness (gameful design).

18 Bien que l'écrasante majorité des exemples actuels de gamification soit numérique, le terme ne saurait être limité à la technologie numérique. Non seulement la convergence des médias et l'omniprésence de l'informatique brouillent de plus en plus la distinction 
entre le numérique et le non-numérique, mais les jeux et le game design sont euxmêmes des catégories transmédiatiques (Juul, 2005).

\section{2 Élément}

19 Alors que "serious game " décrit la conception de jeux à part entière à des fins non divertissantes, les applications gamifiées ne font qu'incorporer des éléments de jeu (ou « atomes » de jeu : cf. Brathwaite \& Schreiber, 2008). Naturellement, la frontière entre un jeu et un artefact avec des éléments de jeu peut souvent être brouillée - Foursquare est-il un jeu ou une application gamifiée ? Pour compliquer les choses, cette frontière est empirique, subjective et socialement construite : le fait de savoir si vous et vos amis «jouez à » ou «utilisez» Foursquare dépend de votre objectif (négocié), de vos perceptions et représentations. L'ajout d'une règle informelle ou d'un objectif partagé par un groupe d'utilisateurs peut transformer une "simple " application gamifiée en un jeu « complet». Dans les études sur le jeu, on reconnaît de plus en plus que toute définition de "games » doit aller au-delà des propriétés de l'artefact ludique pour inclure ces significations situées et socialement construites (Consalvo, 2009; Taylor, 2009). Pour notre propos, cela signifie que (a) l'on doit prendre en compte dans les jeux aussi bien les éléments d'artefact que la dimension sociale, et (b) que les éléments d'artefact devraient être envisagés davantage comme des moyens permettant des interprétations et représentations gameful, plutôt que comme des éléments étant euxmêmes gameful. En effet, la caractéristique des applications gamifiées pourrait consister en ce que, comparées aux jeux, elles procurent un enchaînement d'expériences et de représentations plus fragile, plus instable, entre les aspects playful, gameful et d'autres modalités plus instrumentales et fonctionnalistes.

Cela mène directement à une autre question : quels éléments appartiennent au groupe des éléments de jeu? Considérons les « Dix Ingrédients des grand jeux » identifiés par Reeves et Read (Reeves \& Read, 2009) : la représentation de soi via un avatar; des environnements en trois dimensions; un contexte narratif; du feedback; des réputations, des rangs, et des niveaux ; des marchés et une économie ; une compétition régie par des règles explicites et fermement appliquées; des équipes; des systèmes de communication parallèles qui peuvent être facilement configurés ; un temps limité. On peut trouver chacun de ces éléments en dehors des jeux et, pris isolément, aucun d'entre eux ne serait facilement identifié comme gameful, et encore moins comme spécifique au jeu. Par ailleurs, on observe une sérieuse variation entre les différents genres de jeu et entre jeux numériques et non numériques - les avatars sont communs dans les jeux d'action et dans les jeux de rôles, mais pas nécessairement dans les jeux vidéo de stratégie ou dans les jeux de cartes. De plus, la façon dont les éléments de jeu sont perçus peut aussi dépendre de notre rôle, que l'on soit designer ou utilisateur. Par exemple, le modèle Mechanics, Dynamics, Aesthetics (MDA) (Hunicke, Leblanc \& Zubek, 2004) suggère que les designers travaillent avec des mécanismes pour créer de l'esthétique, alors que les joueurs font l'expérience de l'esthétique et ce, faisant en déduisent un savoir à propos d'une connaissance des mécanismes.

Tout cela met en évidence que "game » est une catégorie composite dont l'existence dépend de multiples conditions nécessaires. Considérons le "modèle classique du jeu " de Juul (Juul, 2005) : « un jeu est un système formel régi par des règles avec un résultat variable et quantifiable, dans lequel différentes valeurs sont attribuées à différents 
résultats, le joueur exerce un effort afin d'influencer le résultat, le joueur se sent concerné par le résultat, et les conséquences de l'activité sont optionnelles et négociables ». Comme Juul lui-même le reconnaît, aucune partie de cette définition ne constitue en elle-même un jeu. Il n'y a qu'ensemble qu'elles dessinent une forme distincte dans l'arrière-plan des autres phénomènes.

Toutefois, aussi utile que ce modèle puisse être pour définir les jeux, il ne dit pas comment identifier les éléments du jeu. Une solution consiste à considérer les éléments de jeu comme un ensemble de briques de construction ou de caractéristiques partagées par les jeux (plutôt que comme un ensemble de conditions nécessaires à un jeu), à la manière des ressemblances familiales de Wittgenstein. Une interprétation très stricte de cette approche - n'accepter que les éléments qui sont uniques ou spécifiques aux jeux - produirait un ensemble vide ou très restreint. Une interprétation très libérale tout élément qui peut être trouvé dans n'importe quel jeu - serait sans limites. De ce fait nous suggérons de restreindre "gamification» à la description des éléments qui sont caractéristiques des jeux - les éléments qui se trouvent dans la plupart des jeux (mais pas nécessairement dans tous), aisément associés aux jeux, et qu'on estime jouer un rôle significatif dans le gameplay. Bien sûr, il s'agit d'une définition heuristique qui laisse une grande latitude pour débattre de ce qui est « caractéristique » des jeux.

\subsection{Design}

Comme nous l'avons vu, les applications gamifiées ne sont pas les seuls cas où des éléments de jeux sont recyclés. En IHM, il existe une longue tradition d'utilisation de contrôleurs de jeu comme périphériques d'entrée à des fins non ludiques. Des moteurs graphiques et des outils de création de jeux vidéo sont également régulièrement utilisés dans des perspectives autres que le divertissement (de la visualisation scientifique et des environnements 3D au fan art), comme c'est le cas pour d'autres pratiques dans le milieu du jeu au sens large, par exemple dans les serious games. Pour plus de clarté sur les plans terminologique et conceptuel, il est plus utile de réserver le terme «gamification » à l'usage du game design et non aux technologies fondées sur le jeu ou aux pratiques du milieu du jeu en général.

Lorsque nous avons passé en revue la littérature existante sur les jeux et la gamification, nous avons constaté que ces éléments de game design étaient identifiés en variant les niveaux d'abstraction. Tous ces niveaux devraient être inclus dans la définition. On peut en distinguer cinq, du plus concret au plus abstrait (cf. tableau 1): les patrons de conception d'interfaces (Crumlish \& Malone, 2009); les patrons de game design (Björk \& Holopainen, 2005) ou les mécanismes de jeu (Taylor, 2009); les principes, l'heuristique ou les "prismes » du design (Schaffer, 2008); les modèles conceptuels des ateliers de game design (Brathwaite \& Schreiber, 2008 ; Calvillo-Gamez, Cairns \& Cox, 2010 ; Fullerton, 2008 ; Hunicke, Leblanc \& Zubek, 2004) ; les méthodes du game design et les procédés de conception (Belman \& Flanagan, 2010 ; Fullerton, 2008).

Tableau 1 : Niveaux des éléments de game design

\begin{tabular}{|l|l|l|}
\hline Niveau & Description & Exemple \\
\hline
\end{tabular}




\begin{tabular}{|c|c|c|}
\hline $\begin{array}{ll}\text { Patrons } & \text { de } \\
\text { conception } & \\
\text { d'interfaces de } \\
\text { jeu }\end{array}$ & $\begin{array}{l}\text { Des éléments de design numérique } \\
\text { courants et reconnus et des solutions de } \\
\text { design adaptées à un problème connu } \\
\text { dans un contexte donné, y compris des } \\
\text { solutions prototypiques }\end{array}$ & Badge, classement, niveau \\
\hline $\begin{array}{l}\text { Patrons de game } \\
\text { design et } \\
\text { mécanismes }\end{array}$ & $\begin{array}{l}\text { La reprise courante des éléments de } \\
\text { design d'un jeu qui concernent le } \\
\text { gameplay }\end{array}$ & $\begin{array}{l}\text { Contrainte de temps, ressources } \\
\text { limitées, tours de jeu }\end{array}$ \\
\hline $\begin{array}{ll}\text { Principes } & \text { et } \\
\text { heuristique } & d u \\
\text { design } & \end{array}$ & $\begin{array}{l}\text { Des principes d'évaluation pour aborder } \\
\text { un problème de design ou analyser une } \\
\text { solution de design donnée }\end{array}$ & $\begin{array}{l}\text { Jouer régulièrement, objectifs précis, } \\
\text { diversité de styles de jeu }\end{array}$ \\
\hline $\begin{array}{l}\text { Modèles du game } \\
\text { design }\end{array}$ & $\begin{array}{l}\text { Modèles conceptuels des composants des } \\
\text { jeux ou de l'expérience de jeu }\end{array}$ & $\begin{array}{l}\text { MDA (Mechanics, Dynamics, Aesthetics); } \\
\text { challenge, fantasy, curiosity; game } \\
\text { design atoms; CEGE (Core Elements of } \\
\text { the Gaming Experience) }\end{array}$ \\
\hline $\begin{array}{l}\text { Méthodes du } \\
\text { game design }\end{array}$ & $\begin{array}{l}\text { Pratiques et procédés spécifiques du } \\
\text { game design }\end{array}$ & $\begin{array}{l}\text { Test des jeux, design centré sur le } \\
\text { play, game design responsable }\end{array}$ \\
\hline
\end{tabular}

Comme on peut le voir, ce "modèle des niveaux » distingue les patrons de conception d'interfaces des patrons de game design ou des mécanismes de jeu. Bien qu'ils soient liés au même concept de pattern languages (Dixon, 2009), contrairement aux patrons de conception d'interfaces, ni les mécanismes de jeu ni les patrons de game design ne renvoient à la mise en œuvre de solutions (prototypiques); les uns comme les autres peuvent être mis en œuvre avec de nombreux éléments d'interface différents. Ils sont donc plus abstraits et de ce fait considérés comme distincts.

Récapitulons : alors que les serious games remplissent toutes les conditions nécessaires et suffisantes pour être des jeux, les applications gamifiées n'empruntent aux jeux que quelques éléments de design. Dans la perspective du designer, ce qui distingue la gamification des jeux « normaux » visant à divertir et des serious games réside dans le fait qu'elle se construit avec l'intention de développer un système qui inclut des éléments des jeux et non un jeu à part entière. Du point de vue de l'utilisateur, de tels systèmes comportant des éléments de design issus des jeux peuvent alors être investis et vécus comme de "vrais jeux ", comme des expériences de type gameful ou playful ou autres cette instabilité ou cette ouverture constituant ce qui les différencie des "vrais jeux » pour les utilisateurs.

\subsection{Des contextes non ludiques}

Comme les serious games, la gamification utilise des éléments de jeux à des fins autres que l'usage normal qui en serait attendu dans le contexte d'un jeu de divertissement. L'« usage normal » est une catégorie socialement, historiquement et culturellement située. Cependant, on peut raisonnablement supposer que le divertissement constitue actuellement le principal horizon d'attente lorsqu'on a recours à des jeux. De même, le plaisir d'utilisation, l'engagement et, de manière plus générale, l'amélioration de 
l'expérience de l'utilisateur représentent actuellement les situations prédominantes d'usage de la gamification (dans la définition proposée dans cet article, les expériences gameful constituent l'objectif de design le plus fréquent). Malgré tout, nous suggérons ouvertement de ne pas délimiter la gamification à des contextes d'usage, des buts ou des scénarios spécifiques. En premier lieu, il n'y a aucun avantage clair à le faire. Deuxièmement, on peut directement relier l'opacité du discours sur les serious games au fait que certains auteurs ont initialement attaché le terme au contexte et à l'objectif spécifiques de l'éducation et de l'apprentissage, alors que les serious games ont proliféré dans toutes sortes de domaines (Sawyer \& Smith, 2001). Par conséquent, parallèlement à la taxonomie des serious games de Sawyer (Sawyer \& Smith, 2001), nous envisageons différents contextes ou domaines d'utilisation comme sous-catégories potentielles : de la même manière qu'il y a des jeux d'entraînement (training games), des jeux sur la santé (health games), des jeux d'information (newsgames), la notion de gameful design ou la gamification peut s'appliquer à l'entraînement, la santé, l'information, ainsi qu'à d'autres domaines d'application.

Certains auteurs ont avancé que les jeux eux-mêmes peuvent être gamifiés (Hamari \& Eranti, 2011) à l'image des plateformes de méta-jeux telles que les systèmes de récompenses (Jakobsson \& Sotamaa, 2011; Montola et al, 2009). En principe, cet argument pourrait aller dans le sens de la définition présentée ici - la seule chose que les « contextes non ludiques » visent explicitement à exclure est l'usage d'éléments de game design dans la conception d'un jeu, puisque nous serions alors simplement en présence de game design et non de gamification. Cependant, à y regarder de plus près, considérer les méta-jeux et autres ajouts de game design à des jeux existants comme relevant d'une autre chose que du game design devient difficile à soutenir : tout d'abord, même dans la littérature formaliste sur le jeu, les méta-jeux sont aussi envisagés comme des jeux à part entière, "basés sur les effets et les résultats d'autres jeux " (Björk \& Holopainen, 2005, p. 401), et pas seulement sur des éléments de game design. Deuxièmement, dans la perspective du designer, étant donné que le contexte du design est déjà celui des jeux, le fait de distinguer le design des méta-jeux (ou des éléments de jeu) de celui de ces jeux semble contreproductif. Troisièmement, si l'on adopte le point de vue de l'utilisateur, savoir si (et dans quelles circonstances) les joueurs perçoivent les éléments du méta-jeu comme relevant du «jeu principal» ou comme lui étant extérieurs est une question empirique complexe qui reste ouverte. Et dans tous les cas où un système de méta-jeu n'est pas perçu comme distinct du jeu «principal », la création d'une séparation artificielle apparaît superflue. Enfin, nous avons soutenu qu'une partie de l'originalité et de la spécificité des systèmes gamifiés réside dans l'enchaînement d'expériences entre les modes gameful, playful et d'autres modes d'expérience et d'engagement. De tels enchaînements d'expérience ont probablement moins de chance de se produire quand l'utilisateur est déjà en train de jouer à un jeu. Qualifier les méta-jeux de "gamification" ne légitime pas cette différence, mais nous admettons volontiers qu'il s'agit d'un cas complexe qui demande de nouvelles recherches empiriques.

\section{Situer la gamification}

En résumé, la gamification renvoie à :

- L'usage (plutôt que l'extension) 
- d'éléments (plutôt que de jeux à part entière)

- de design (plutôt que de technologies fondées sur le jeu ou d'autres pratiques liées au jeu)

- caractéristiques des games (plutôt que du play ou playfulness)

- dans des contextes non ludiques (quel que soit le but spécifique de leur utilisation, le contexte, ou le média qui les met en œuvre).

Cette définition met en opposition la gamification et d'autres concepts apparentés à travers les deux distinctions suivantes : playing/gaming et éléments/ensemble. Les jeux et les serious games peuvent tous deux être différenciés de la gamification en ce que les premiers forment des ensembles, alors que la seconde emprunte des éléments à ces ensembles. Le playful design et les jouets peuvent se distinguer sur l'axe playing/gaming (schéma 1).

Schéma 1. La gamification sur les axes playing/gaming et éléments/ensemble

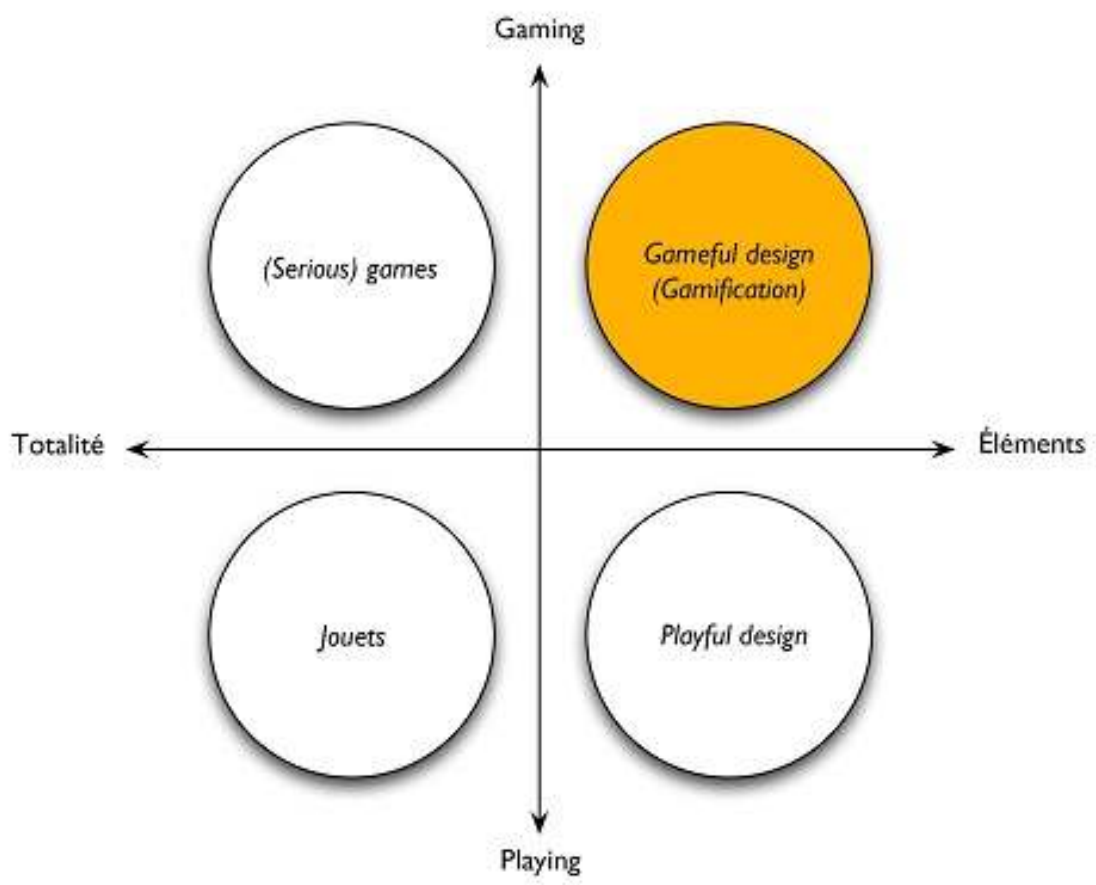

Dans le schéma général de tendances et de concepts qui apparaissent en relation, la gamification ou le gameful design se situe de la manière suivante : dans la tendance socioculturelle de la ludification, au moins trois dynamiques se rapportent aux jeux vidéo et à l'IHM : l'extension des jeux (les jeux pervasifs), l'utilisation de jeux dans des contextes non ludiques, et l'interaction playful. L'utilisation de jeux dans des contextes non ludiques s'applique à des jeux à part entière (serious games) et à des éléments de jeu, que l'on peut diviser en technologies, pratiques et game design. Ce dernier élément renvoie à la gamification (schéma 2). 


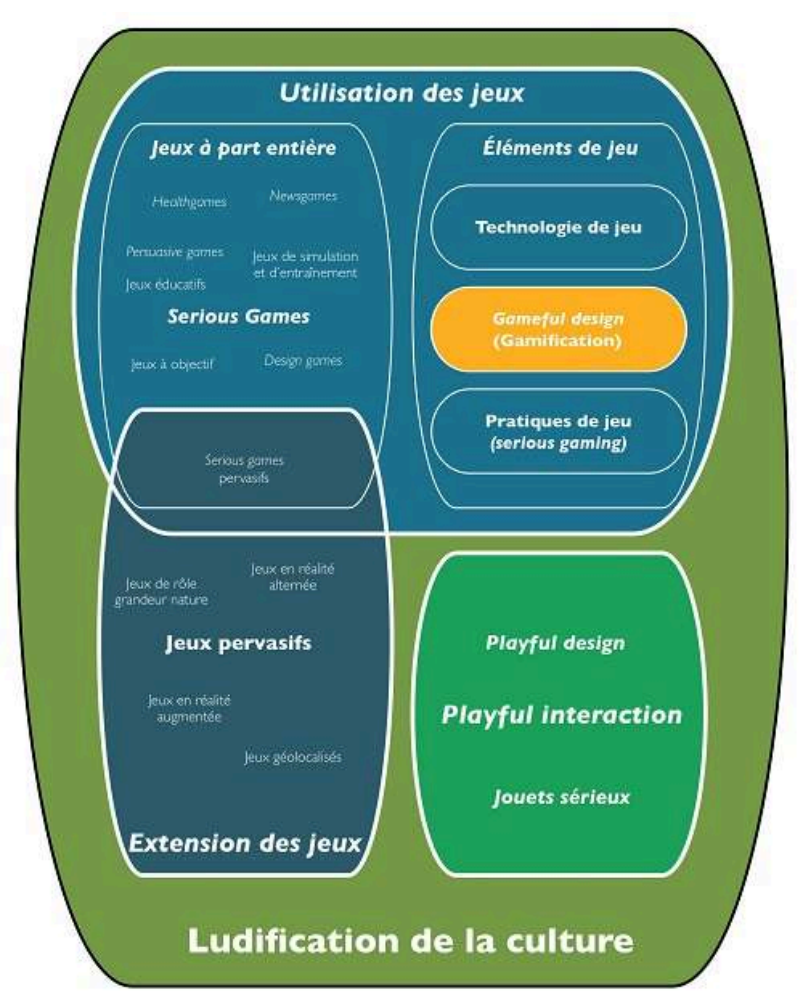

32 À ce jour, il semble qu'il n'y a eu qu'une seule autre tentative de définition de "gamification» dans la littérature scientifique. Huotari et Hamari ont proposé de définir "gamification» dans une perspective de markéting des services, comme « une gamme de services dans laquelle un service principal est amélioré par un système de services régi par des règles qui permet à l'utilisateur de bénéficier de mécanismes de réponse et d'interaction dans le but de faciliter et de soutenir la création de valeur ajoutée pour les utilisateurs » (Huotari \& Hamari, 2011).

La définition de Huotari et Hamari diffère de la nôtre en plusieurs points. Tout d'abord, puisqu'elle se focalise sur les systèmes régis par des règles, on peut soutenir qu'elle s'étend au-delà des jeux et des services gamifiés et qu'elle peut s'appliquer en fin de compte à presque tout système interactif. Même un écran tactile pour commander des friandises dans un cinéma remplit les conditions d'un « système de services régi par des règles » (mû par un logiciel) « qui permet à l'utilisateur de bénéficier de mécanismes de réponse et d'interaction" (les gens commandent via l'interface, qui confirme leurs commandes), « dans le but de faciliter et de soutenir la création de valeur ajoutée pour les utilisateurs » (la capacité de commander des friandises améliore l'expérience du film).

En deuxième lieu, le fait de se focaliser sur les systèmes régis par des règles et de situer la définition dans la perspective du markéting de services minimise les dimensions sociales et expérientielles constitutives du jeu.

Troisièmement, la définition exclut tous les systèmes dont la mise à disposition de mécanismes de jeu (adaptés à un contexte spécifique) constitue en elle-même le service de base ou tout le moins une partie essentielle de celui-ci : ce que proposent la plupart des applications de santé gamifiées, telles que Health Month (healthmonth.com), c'est la capacité d'établir pour une personne des règles et des objectifs en matière de 
comportement sanitaire, puis de suivre les comportements effectifs qui vont à leur encontre - ces éléments de jeu ne sont pas une " amélioration » déductible d'un autre service « de base ». A contrario, nous pensons que notre définition aborde toutes ces questions.

\section{Conclusion}

Dans cet article, nous avons soutenu que les applications gamifiées actuelles donnent à voir des phénomènes émergents qui justifient de nouveaux concepts et de nouvelles recherches. Plus spécifiquement, nous avons suggéré que l'analyse de la notion de «gamefulness » en complément de la notion de «playfulness »- en termes d'objectifs de conception comme de comportements et d'expériences d'utilisateur - constitue une contribution précieuse et durable dans l'étude des systèmes gamifiés. Nous avons aussi introduit le terme "gameful design » - le design pour des expériences de type gameful comme une alternative possible à "gamification». Au regard des origines industrielles, des fortes connotations et des débats autour de la pratique et de la conception de la gamification, "gameful design » permet de disposer d'un nouveau terme moins chargé et, en conséquence, plus adapté à la discussion scientifique.

Le fort degré de subjectivité et la variabilité du contexte lorsqu'on veut identifier la gamification constituent deux autres points importants. Il n'est pas possible de déterminer si un système empirique donné est «une application gamifiée » ou «un jeu » sans recourir soit aux intentions du designer soit aux expériences et aux représentations des utilisateurs. En effet, comparées aux jeux d'une part et aux logiciels utilitaires d'autre part, les applications gamifiées présentent la particularité d'être relativement enclines à faire varier les modes situationnels d'engagement - gameful, playful et instrumental.

En conclusion, l'une des grandes promesses du déploiement commercial actuel de systèmes gamifiés est de permettre d'accéder facilement à des données d'utilisateurs plus pertinentes quant aux différents types d'expériences et de catégories naturelles qui émergent de l'interaction avec ces systèmes. Ces données détermineront au final la validité des distinctions que nous avons opérées ici. Même si elles se révélaient impossibles à soutenir sur le long terme, nous pensons que les définitions de "gamification» et de "gamefulness» que nous avons proposées contre les serious games et l'interaction de type playful clarifient le débat et permettent donc à la recherche d'évoluer vers des études plus pointues et vers une conceptualisation plus nette des phénomènes définis.

\section{BIBLIOGRAPHIE}

ABT C.C. (1970), Serious Games, New York, Viking. 
AHN L. von \& DABBISH L. (2008), « Designing games with a Purpose », Communications of the ACM, vol. 51, n 8, pp. 58-67.

ALFRINK K. (2011), « New games for new cities », communication au colloque «FutureEverything », Manchester, en ligne: http://goo.gl/6Wfx4

BARR P. (2007), Video Game Values: Play as Human-Computer Interaction, Thèse de doctorat, Victoria University of Wellington.

BELMAN J. \& FLANAGAN M. (2010), « Exploring the Creative Potential of Values Conscious Game Design: Students' Experiences with the VAP Curriculum », Eludamos, vol. 4, n 1, pp. 57-67, en ligne: http://www.eludamos.org/index.php/eludamos/article/view/vol4no1-5/156

BERNHAUPT R. (dir.) (2010), Evaluating User Experience in Games: Concepts and Methods, London, Springer.

BJÖRK S. \& HOLOPAINEN J. (2005), Patterns in Game Design, Boston, Charles River Media.

BLYTHE M., HASSENZAHL M. \& WRIGHT P. (2004), «Introduction », Interactions, vol. 11, n 5 , pp. 36-37.

BOGOST I. (2011), « Persuasive Games : Exploitationware », Gamasutra.com, en ligne :

BRATHWAITE B. \& SCHREIBER I. (2008), Challenges for Game Designers, Boston, Charles River Media.

BURGHARDT G.M. (2005), The Genesis of Animal Play: Testing the Limits, Cambridge, MIT Press.

CAILLOIS R. (1958), Les jeux et les hommes, Paris, Gallimard.

CALVILLO-GAMEZ E.H., CAIRNS P. \& COX A.L. (2010), « Assessing the Core Elements of the Gaming Experience », in Bernhaupt R. (dir.), Evaluating User Experience in Games: Concepts and Methods, London, Springer, pp. 47-71.

CARROLL J.M. (1982), « The Adventure of Getting to Know a Computer », Computer, vol. 15, $\mathrm{n}^{\circ} 11$, pp. 49-58.

CARROLL J.M. \& THOMAS J.C. (1982), « Metaphor and the Cognitive Representation of Computing Systems », IEEE Transactions on Systems, Man, and Cybernetics, 12, pp. 107-116.

CARROLL J.M. \& THOMAS J.C. (1988), « FUN », ACM SIGCHI Bulletin, vol. 19, n 3, pp. 21-24.

CHAO D. (2001), « Doom as an Interface for Process Management », CHI 01 Proceedings of the SIGCHI Conference on Human Factors in Computing Systems, New York, ACM Press, pp. 152-157.

CHATFIELD T. (2005), Fun Inc.: Why Gaming Will Dominate the Twenty-First Century, London, Pegasus.

CONSALVO M. (2009), « There is No Magic Circle », Games and Culture, vol. 4, n 4, pp. 408-417.

COSTELLO B. \& EDMONDS E. (2007), « A Study in Play, Pleasure and Interaction Design », DPPI 07 Proceedings of the 2007 Conference on Designing Pleasurable Products and Interfaces, New York, ACM Press, pp. 76-91.

CRUMLISH C. \& MALONE E. (2009), Designing Social Interfaces: Principles, Patterns, and Practices for Improving the User Experience. Sebastopol, O'Reilly.

CSIKSZENTMIHALYI M. (1990), Flow: the Psychology of Optimal Experience, New York, Harper and Row.

Designing for Ludic Engagement », CHI '04 Extended Abstracts on Human Factors in Computing Systems, New York, ACM Press, pp. 885-900. 
DETERDING S. (2012), « Playful Technologies », in Wiedemann C. and Zehle S. (dir.), Depletion Design. A Glossary of Network Ecologies, Amsterdam, Institute of Network Cultures, pp. 121-126. DETERDING S., SICART M., NACKE L., O'HARA K. \& DIXON D. (2011), « Gamification: Using gamedesign elements in non-gaming contexts », CHI '11 Extended Abstracts on Human Factors in Computing Systems, New York, ACM Press, pp. 2425-2428.

DIGNAN A. (2011), Game Frame: Using Games as a Strategy for Success, New York, Free Press. DIXON D. (2009), « Pattern Languages for CMC Design », in Whitworth B. and De Moor A. (dir.), Handbook of Research on Socio-Technical Design and Social Networking Systems, Hershey, IGI Global, pp. 402-415.

FERRARA J. (2012), Playful Design. Creating Game Experiences in Everyday Interfaces, New York, Rosenfeld Media.

FLATLA D.R., GUTWIN C., NACKE L.E., BATEMAN S. \& MANDRYK R.L. (2011), « Calibration Games: Making Calibration Tasks Enjoyable by Adding Motivating Game Elements », UIST '11: Proceedings of the 24th Annual ACM Symposium on User Interface Software and Technology, ACM Press, pp. 403-412.

FONTIJN W. \& HOONHOUT J. (2007), « Functional Fun with Tangible User Interfaces », Proceedings of DIGITEL 2007 The First IEEE International Workshop on Digital Game and Intelligent Toy Enhanced Learning, pp. 119-123.

FULLERTON T. (2008), Game Design Workshop: A Playcentric Approach to Creating Innovative Games, Amsterdam, Morgan Kaufmann.

GAVER W. W., BOWERS J., BOUCHER A., GELLERSON H., PENNINGTON S., SCHMIDT A., STEED A., VILLARS N. \& WALKER B. (2004), « The Drift Table: Designing for Ludic Engagement”, CHI '04 Extended Abstracts on Human Factors in Computing Systems, New York, ACM Press, pp. 885-900 GAVER W.W. (2002), « Designing for Homo Ludens », I3 Magazine, $\mathrm{n}^{\circ} 12$.

GRACE M.V. \& HALL J. (2008), « Projecting Surveillance Entertainment », Communication au colloque « Emerging Technology Conference », San Diego.

HALTER E. (2006), From Sun Tzu to Xbox: War and Videogames, New York, Thunder's Mouth Press.

HAMARI J. \& ERANTI V. (2011), « Framework for Designing and Evaluating Game Achievements », Proceedings of the Fifth International Conference of the Digital Research Association (DIGRA): Think Design Play, en ligne: http://www.digra.org/wp-content/uploads/digital-library/ 11307.59151.pdf

HASSENZAHL M. (2003), « The Thing and I: Understanding the Relationship Between User and Product », in Blythe M.A., Overbeeke K., Monk A.F. \& Wright P.C. (dir.), Funology: From Usability to Enjoyment, New York, Kluwer, pp. 31-41.

HELGASON D. (2010), « 2010 Trends », unity3d.com, en ligne : http://goo.gl/AZ4vm http://gamification-research.org/wp-content/uploads/2011/04/14-Huotari.pdf http://goo.gl/jK1VR.

http://hci.usask.ca/uploads/214-UIST-2011.pdf

HUNICKE R., LEBLANC M. \& ZUBEK R. (2004), « MDA: A Formal Approach to Game Design and Game Research », Proceedings of the AAAI Workshop on Challenges in Game AI, AAAI Press. 
HUOTARI K. \& HAMARI J. (2011), « Gamification from the Perspective of Service Marketing », Proceedings of the CHI 2011 Workshop Gamification.

JAKOBSSON M. \& SOTAMAA O. (2011), « Special Issue -Game Reward Systems », Game Studies, vol. 11, n 1, en ligne: http://gamestudies.org/1101/articles/editorial_game_reward_systems JENKINS H., CAMPER B., CHISHOLM A., GRIGSBY N., KLOPFER E., OSTERWEIL S., PERRY J., TAN P., WEISE M. \& CHOR GUAN T. (2009), « From Serious Games to Serious Gaming », in Ritterfeld U., Cody M. and Vorderer P. (dir.), Serious Games: Mechanisms and Effects, New York, Routledge, pp. 448-468.

JORDAN P.W. (2002), Designing Pleasurable Products. An Introduction to the New Human Factors, London, New York, Taylor and Francis.

JUUL J. (2005), Half-Real: Video Games Between Real Rules and Fictional Worlds, Cambridge, MIT Press.

JUUL J. (2010), A Casual Revolution: Reinventing Video Games and Their Players, Cambridge, MIT Press.

KORHONEN H., MONTOLA M. \& ARRASVUORI J. (2009), « Understanding Playful User Experiences Through Digital Games », DPPI 2009 Proceedings of the International Conference on Designing Pleasurable Products and Interfaces, ACM Press, pp. 274-285.

LUCERO A. \& ARRASVUORI J. (2010), « PLEX Cards: a Source of Inspiration when Designing for Playfulness », Fun and Games '10 Proceedings of the 3rd International Conference on Fun and Games, ACM Press, pp. 28-37.

MALABY T.M. (2007), « Beyond Play: A New Approach to Games », Games and Culture, vol. 2, n 2, pp. 95-113.

MALONE T.W. (1981), « Toward a Theory of Intrinsically Motivating Instruction », Cognitive Science, $\mathrm{n}^{\circ} 4$, pp. 333-370.

McDONALD M., MUSSON R. \& SMITH R. (2008), « Using Productivity Games to Prevent Defects », in McDonald M., Musson,R. and Smith R. (dir.), The Practical Guide to Defect Prevention, Redmond, Microsoft Press, pp. 79-95.

McGONIGAL J. (2011), Reality Is Broken: Why Games Make Us Better and How They Can Change the World, London, Penguin.

MONTOLA M., NUMMENMAA T., LUCERO A., BOBERG M. \& KORHONEN H. (2009), « Applying Game Achievement Systems to Enhance User Experience in a Photo Sharing Service ", Proceedings of the 13th International MindTrek Conference: Everyday Life in the Ubiquitous Era on - MindTrek '09, ACM Press, pp. 94-97.

MONTOLA M., STENROS J. \& WAERN A. (2009), Pervasive Games: Theory and Design. Experiences on the Boundary Between Life and Play, Amsterdam, Morgan Kaufmann.

MORRISON A., VILLER S. \& MITCHELL P. (2011), « Building Sensitising Terms to Understand FreePlay in Open-Ended Interactive Art Environments », CHI 11 Proceedings of the SIGCHI Conference on Human Factors in Computing Systems, ACM Press, pp. 2335-2344.

MORRISON A.J., MITCHELL P. \& BRERETON M. (2007), « The Lens of Ludic Engagement », MULTIMEDIA '07 Proceedings of the 15th International Conference on Multimedia, ACM Press, pp. 509-512.

NACKE L.E., DRACHEN A. \& GOEBEL S. (2010), « Methods for Evaluating Gameplay Experience in a Serious Gaming Context », International Journal of Computer Science in Sport, vol. 9, $\mathrm{n}^{\circ} 2$. 
PAHARIA R. (2010), réponse à la question « Who coined the term "gamification" ? " posée sur le site quora.com, en ligne : http://goo.gl/CvcMs

PAHARIA R. (2011), communication du 17 mars 2011.

PRIEBATSCH S. (2011), « The Game Layer on Top of the World », Communication à la conférence SxSWi, Austin.

RAESSENS J. (2006), «Playful Identities, or the Ludification of Culture », Games and Culture, vol. $1, \mathrm{n}^{\circ} 1$, pp. 52-57.

REEVES B. \& READ J.L. (2009), Total Engagement: Using Games and Virtual Worlds to Change the Way People Work and Businesses Compete, Boston, Harvard Business School Press.

RITTERFELD U., CODY M. \& VORDERER P. (2009), Serious Games: Mechanisms and Effects, London, Routledge.

SALEN K. \& ZIMMERMAN E. (2004), Rules of Play: Game Design Fundamentals, Cambridge, MIT Press.

SAWYER B. \& SMITH P. (2008), « Serious Games Taxonomy », Communication à la Games Developers Conference, San Francisco.

SCHAFFER N. (2008), « Heuristic Evaluation of Games », in Isbister K. \& Schaffer N. (dir.), Game Usability: Advice from the Experts for Advancing the Player Experience, Amsterdam, Morgan Kaufman, pp. 79-89.

SCHELL J. (2010), «Visions of the Gamepocalypse », Communication à la Long Now Foundation, San Francisco.

SICART M. (2008), « Defining Game Mechanics », Game Studies, vol. 8, n² 2, en ligne: http:// gamestudies.org/0802/articles/sicart

SWEETSER P. \& WYETH P. (2005), « GameFlow: A Model for Evaluating Player Enjoyment in Games », Computers in Entertainment, vol. 3, $\mathrm{n}^{\circ} 3$.

TAKAHASHI D. (2008), « Funware's Threat to the Traditional Video Game Industry », venturebeat.com, en ligne: http://goo.gl/o9lsq

TAYLOR T.L. (2009), « The Assemblage of Play », Games and Culture, vol. 4, n 4, pp. 331-339.

WOSZCZYNSKI A.B., ROTH P.L. \& SEGARS A.H. (2002), « Exploring the Theoretical Foundations of Playfulness in Computer Interactions ", Computers in Human Behavior, vol. 18, $n^{\circ} 4$, pp. 369-388.

ZHANG P. (2008), « Motivational Affordances: Reasons for ICT Design and Use », Communications of the ACM, vol. 51, $\mathrm{n}^{\circ} 11$, pp. 145-147.

ZICHERMANN G. (2011), « A Long Engagement and a Shotgun Wedding: Why Engagement is the Power Metric of the Decade », communication au Gamification Summit, San Francisco, en ligne: http://goo.gl/jlaOo

ZICHERMANN G. \& CUNNINGHAM C. (2011), Gamification by Design: Implementing Game Mechanics in Web and Mobile Apps, Sebastopol, O’Reilly.

\section{NOTES}

1. http://www.bunchball.com/nitro/ 
2. Il y a un certain consensus sur le fait que la notion de playfulness doit être interprétée comme une attitude ou un état d'esprit avec lequel on approche une activité donnée, plutôt que comme un ensemble distinct de comportements observables. Cependant, plusieurs chercheurs font aussi remarquer qu'il y a toujours certaines propriétés formelles observables lors d'activités lorsqu'elles relèvent d'une intention ludique (Burghardt, 2005 ; Caillois, 2001; Malaby, 2007). Pour rendre compte de cela, nous parlons ici d' « attitude et d'état d'esprit ».

\section{RÉSUMÉS}

Ces dernières années, on a assisté à une rapide prolifération sur le marché de la grande consommation de logiciels qui s'inspirent des jeux vidéo. Habituellement qualifiée de "gamification", cette tendance est liée à un vaste ensemble de concepts et de recherches existant dans le champ des interactions homme-machine et des études sur le jeu, tels que les serious games, les jeux pervasifs, les jeux en réalité alternée, ou le playful design. Cependant, le rapport qu'entretient la " gamification » avec ces éléments, la question de savoir si elle constitue un phénomène nouveau et la façon de la définir ne sont pas clairs. C'est pourquoi nous étudions dans cet article la "gamification » et les origines historiques du terme en lien avec des termes précurseurs et des concepts similaires. Nous avançons l'idée selon laquelle les applications "gamifiées" donnent à voir de nouveaux phénomènes que l'on nommera gameful, complémentaires des phénomènes playful. En nous appuyant sur notre recherche, nous proposons de définir la gamification comme «l'usage d'éléments de game design dans des contextes non ludiques ».

Recent years have seen a rapid proliferation of mass-market consumer software that takes inspiration from video games. Usually summarized as "gamification", this trend connects to a sizeable body of existing concepts and research in human-computer interaction and game studies, such as serious games, pervasive games, alternate reality games, or playful design. However, it is not clear how "gamification" relates to these, whether it denotes a novel phenomenon, and how to define it. Thus, in this paper we investigate "gamification" and the historical origins of the term in relation to precursors and similar concepts. It is suggested that "gamified" applications provide insight into novel, gameful phenomena complementary to playful phenomena. Based on our research, we propose a definition of "gamification" as the use of game design elements in non-game contexts.

\section{INDEX}

Keywords : alternate reality games, game-based technologies, gameful design, gamefulness, games, gamification, pervasive games, play, playful design, playfulness, serious games

Mots-clés : jeux en réalité alternée, technologies fondées sur le jeu, gameful design, gamefulness, games, gamification, jeux pervasifs, play, playful design, playfulness, serious games 


\section{AUTEURS}

\section{SEBASTIEN DETERDING}

Hans Bredow Institute dor Media Research, Université de Hambourg

DAN DIXON

Digital Cultures Research Centre, University of the West of England

\section{RILLA KHALED}

Center for Computer Games Research, IT University of Copenhagen

\section{LENNART NACKE}

Faculty of Business and Information Technology, University of Ontario Institute of Technology 